\title{
Infinitesimal relative position vector fields for observers in a reference frame and applications to conformally stationary spacetimes
}

\author{
Magdalena Caballero ${ }^{\dagger}$, Daniel de la Fuente ${ }^{\star}$ and Rafael M. Rubio ${ }^{\dagger}$ * \\ † Departamento de Matemáticas, \\ Universidad de Córdoba, 14071 Córdoba, Spain \\ E-mails: magdalena.caballero@uco.es, rmrubio@uco.es \\ * Departamento de Matemáticas, \\ Universidad de Oviedo, 33003 Gijón, Spain \\ E-mail: fuentedaniel@uniovi.es
}

\begin{abstract}
We introduce and analyze the concept of infinitesimal relative position vector field between "infinitesimally nearby" observers, showing the equivalence between different definitions. Through the Fermi-Walker derivative of infinitesimal relative position vector fields along an observer in a reference frame, we characterize spacetimes admitting an umbilic foliation. Sufficient and necessary conditions for those spacetimes to be a conformally stationary spacetime are given. Finally, the important class of cosmological models known as generalized Robertson-Walker spacetimes is characterized.
\end{abstract}

Keywords: irrotational vector fields, conformal Killing vector fields, spatially conformal Killing vector fields, observers, fields of observers.

MSC 2010: 53C50, 53B50, 83C99.

\section{Introduction}

The concept of observer has had an important role in the history of physics. Nevertheless, in the case of general relativity with its intrinsic general covariance, the false belief that all observers are physically equivalent has sometimes been developed. As a consequence of this error, the concept of observer has been deprived of part of its meaning, i.e., if we think that all observers are physically equivalent, this concept is irrelevant in the description of a physical scenario.

The reality is that the covariant character of general relativity allows its fundamental equations to hold for any observer. In the case of several relativistic models, the description of the physical scenario is jointed to the election of a distinguished congruence of observers. For example, the Friedmann Robertson-Walker model, whose fiber is flat, represents a perfect

\footnotetext{
${ }^{*}$ The authors are partially supported by Spanish MINECO and FEDER project MTM2016-78807-C2-1-P.
} 
fluid solution for the called comoving observers, which are at rest with respect to the molecules of the perfect fluid. But, this model can also be an exact solution of the field equation for a viscous fluid, with or without an electromagnetic field, as seen by observers moving relative to the previously mentioned comoving observers. Both solutions are physically acceptable (see $[5])$.

In relativistic spacetimes, each observer can establish local coordinates around him, via the exponential map. However, they cannot either extract measurable quantities from a test particle, or compare frame dependent information with another observer, unless they meet at the same point or come close enough so that the spacetime can be considered, effectively, as flat, and then a special relativity like situation is recovered. In general relativity, the Lorentz transformation between two frames is only possible locally. This is quite a different situation than what happens in special relativity. And indeed, this limits the role of the notion of observer in general relativity and cosmology. That is the reason why in general relativity the meaningful physical quantities are those which are observer independent, like the Lorentzian metric, the proper time and the other tensorial quantities as the electromagnetic strength tensor F, etc. Nevertheless, this is quite a realistic situation because real life observers can only measure physical quantities locally. The measurable quantities such as the electric field strength, the magnetic field strength, and in general the energy-momentum tensor are local quantities.

The different nature between observers from special to general relativity is a purely mathematical question, since a differentiable manifold, in general, do not have vector space structure. The position vector and hence the coordinates associated to it, only make sense in a vector space. In a general differentiable manifold, to talk about coordinates we need to focus on a localized region of the manifold, a chart, diffeomorphic to $\mathbb{R}^{n}$, being $n$ the dimension of the manifold. Thus, in general, observes cannot set up reference frames which explore the whole spacetime, making the role of observer strongly local since it can only measure local observations.

Despite the previous comments on observers, global inferences are not impossible. The key here is the concept of symmetry. If the quantities which we are interested in follow a pattern, then the whole spacetime needs not be explored. A study over a local region can be extrapolated to figure out the global structure of the spacetime.

More precisely, in general relativity symmetry is usually based on the assumption of the existence of a one-parameter group of transformations generated by a Killing or, more generally, a conformal Killing vector field. From the information of the metric tensor, one can also find out, if possible, the conformal space transformation and study a great deal about the global properties of spacetime. In fact, an usual simplification for the search of exact solutions to the Einstein equation is to assume the existence a priori of such an infinitesimal symmetry (see $[6,8]$ for instance). A complete general approach to symmetries in general relativity can be found in [22] (see also [7] and references therein). Although the same causal character for the infinitesimal symmetry is not always assumed, the timelike choice is natural, since the integral curves of such a timelike infinitesimal symmetry provide a privileged class of observers or test particles in the spacetime. Moreover, this choice is supported by very well-known examples of exact solutions, [19].

A spacetime $M$ admitting a timelike Killing vector field is called stationary. It can be easily seen that if a spacetime $\mathrm{M}$ has a timelike conformal Killing vector field, then it is globally conformal to a stationary spacetime. This is a reason to call $M$ conformally stationary (CS). Clearly, a CS spacetime is time orientable. In general, the orthogonal distribution defined by 
a timelike conformal Killing vector field $\mathrm{K}$ in a spacetime is not integrable. The necessary and sufficient condition for a vector field to have an integrable orthogonal distribution is to be irrotational (vorticity-free). In particular, if the 1-form metrically equivalent to the vector field is closed, or equivalently, if the vector field is locally the gradient of some function, then its orthogonal distribution is integrable and provides the spacetime with a distinguished foliation by spacelike hypersurfaces. The presence of such a vector field is not enough to prevent the existence of closed non spacelike curves. However, if the timelike vector field $\mathrm{K}$ is globally the gradient of some smooth function ( $\mathrm{K}$ is then called a gradient vector field), then the (clearly non compact) spacetime admits a global time function. Therefore, it is stably causal [11], i.e. there is a fine $C^{0}$ neighborhood of the original metric of the spacetime such that any of its Lorentzian metrics is causal [2]. The existence of a gradient conformal Killing vector field in a spacetime has been used to study certain cosmological models [16] and plays a relevant role for vacuum and perfect fluid spacetimes, see [6]. Spacetimes admitting a timelike gradient conformal Killing vector field (GCS) are then causal (CS spacetimes fail to be causal in general), and they admit an umbilical synchronization, i.e., a foliation by spacelike totally umbilic hypersurfaces. These spacetimes have been widely studied in [3].

From the perspective of the kinetic relativistic theory, CS spacetimes have been characterized as those spacetimes admitting an observer which sees an isotropic microwave background (MWB) and which satisfy the Liouville equation (the Boltzmann equation with null collision term), see [9, Lemma 2]. The non null collision case has been studied in [20], where the authors proved that in a solution of the Einstein-Boltzmann equations with an isotropic distribution function, the velocity is shear-free (spatially conformal Killing) and the product of the expansion and the vorticity vanishes. Therefore, the study of spacetimes with an umbilic synchronization is fully justified. In [9], the Einstein equation for those spacetimes is written, developing a framework suited to obtain inhomogeneous solutions of the Einstein equations compatible with isotropic radiation. From a geometric point of view, those spacetimes are also interesting nowadays, [13].

It is known that small deviations form homogeneity at decoupling time are necessary in order to understand the formation of the observed structures. A privileged subclass of inhomogeneous spacetimes admitting an isotropic radiation, which in addition are GCS spacetimes, is the family of Generalized Robertson-Walker (GRW) spacetimes. Taking into account the results in [8], this class of physically realistic spacetimes is not too much wider. A GRW spacetime is a warped product with base a negatively defined line, fiber a general Riemannian manifold, and arbitrary warping function. Note that, in this definition, the fiber is not assumed to be of constant sectional curvature. When this assumption holds and the dimension of the spacetime is 4, the GRW spacetime is a (classical) Robertson-Walker spacetime. Thus, GRW spacetimes widely extend Robertson-Walker spacetimes, and they include, for instance, the Einstein-de Sitter spacetime, Friedmann cosmological models, the static Einstein spacetime and the de Sitter spacetime. Observe that conformal changes of the metric of a GRW spacetime, with a conformal factor which only depends on universal time, produce new GRW spacetimes. Moreover, small deformations of the metric on the fiber of RobertsonWalker spacetimes also fit into the class of GRW spacetimes. Thus, GRW spacetimes could be suitable spacetimes to model universes with inhomogeneous spacelike geometry [15].

In this work we study two different ways to establish in a accurate mathematical form the concept of infinitesimal relative position vector field with respect to a fixed observer in a congruence or vector field of observers (Section 3). Moreover, we show its mathematical equivalence (see Theorem 4). The change of position that this observer measures for other 
nearby observer is given mathematically thanks to the Fermi-Walker derivative. At this point, we ask ourselves when the observer measures that the nearby observers (for a given congruence), do not rotate. It is clear that this situation holds when the Fermi-Walker derivative of the infinitesimal relative vector fields is collinear to these vector fields. In Proposition 9, we characterize this condition via the Lie derivative of the metric and Curl tensors. As a consequence of this characterization it follows that, in a conformally stationary spacetime whose conformal Killing vector field is irrotational, the position vector fields established by the observers determined by the timelike conformal Killing vector field satisfy the collinearity condition.

A natural mathematical question arises. If the collinearity condition holds associated to a field of observers, when will the spacetime be conformally stationary? This question is widely studied in Theorem 10 and Theorem 11.

Finally, we apply our results to get sufficient conditions under which a spacetime with an umbilic congruence is a GRW spacetime, characterizing this relevant class of cosmological models (see Theorem 14).

\section{Preliminaries: relativistic spacetimes, synchronizability and Fermi-Walker connection}

A relativistic spacetime is an oriented $(n+1)$-dimensional Lorentzian manifold $(M, g), n \geq 1$, endowed with a fixed time orientation, [14]. Along this paper the signature of a Lorentzian metric is considered to be $(-,+, \ldots,+)$. The points of $M$ are also named events. A tangent vector $v \in T_{p} M$ is named spacelike if $g(v, v)>0$ or $v=0$, timelike if $g(v, v)<0$ and lightlike otherwise. A hypersurface in $M$ is called spacelike if its tangent vectors are spacelike, i.e., the induced tensor metric from $(M, g)$ is Riemannian. An observer in $M$ is mathematically represented by a (smooth) curve $\gamma: I \subseteq \mathbb{R} \longrightarrow M$ such that its velocity $\gamma^{\prime}(t)$ is future pointing and $g\left(\gamma^{\prime}(t), \gamma^{\prime}(t)\right)=-1$ for any $t \in I$, (see [17]). The parameter $t$ is called the proper time of the observer.

The Levi-Civita connection $\nabla$ of $(M, g)$, induces a connection along the observer $\gamma$, such that its corresponding covariant derivative is given by $\frac{D Y}{d t}=\nabla_{\gamma^{\prime}(t)} Y \in \mathfrak{X}(\gamma)$ for $Y \in \mathfrak{X}(\gamma)$, where $\mathfrak{X}(\gamma)$ denotes the space of smooth vector fields along $\gamma$. The covariant derivative $\frac{D \gamma^{\prime}}{d t}$ of $\gamma^{\prime}$, is understood as the (proper) acceleration of the observer $\gamma$. When $\frac{D \gamma^{\prime}}{d t} \equiv 0, \gamma$ is a timelike geodesic in $M$, and the observer $\gamma$ is in free falling.

At each event $\gamma(t)$ the tangent space $T_{\gamma(t)} M$ splits as

$$
T_{\gamma(t)} M=T_{t} \oplus R_{t},
$$

where $T_{t}=\operatorname{Span}\left\{\gamma^{\prime}(t)\right\}$ and $R_{t}=T_{t}^{\perp}$. Endowed with the restriction of $g, R_{t}$ is a spacelike hyperplane of $T_{\gamma(t)} M$. It is interpreted as the instantaneous physical space observed by $\gamma$ at $t$ and it is called the infinitesimal rest space of the observer at $\gamma(t)$, see [14]. Clearly, the observer $\gamma$ is able to compare spatial directions at $t$. In order to compare $v_{1} \in R_{t_{1}}$ with $v_{2} \in R_{t_{2}}, t_{1}<t_{2}$ and $\left|v_{1}\right|=\left|v_{2}\right|$, the observer $\gamma$ could use, as a first attempt, the parallel transport along $\gamma$ defined by the Levi-Civita covariant derivative along $\gamma$,

$$
P_{t_{1}, t_{2}}^{\gamma}: T_{\gamma\left(t_{1}\right)} M \longrightarrow T_{\gamma\left(t_{2}\right)} M .
$$


This linear isometry satisfies $P_{t_{1}, t_{2}}^{\gamma}\left(R_{t_{1}}\right)=R_{t_{2}}$ if $\gamma$ is in free falling (i.e., it has null proper acceleration). But, unfortunately, this property does not remain true for any general observer. In order to solve this difficulty, for each $Y \in \mathfrak{X}(\gamma)$ put $Y_{t}^{T}, Y_{t}^{R}$ the orthogonal projections of $Y_{t}$ on $T_{t}$ and $R_{t}$, respectively, i.e., $Y_{t}^{T}=-g\left(Y_{t}, \gamma^{\prime}(t)\right) \gamma^{\prime}(t)$ and $Y_{t}^{R}=Y_{t}-Y_{t}^{T}$. In this way, we define $Y^{T}, Y^{R} \in \mathfrak{X}(\gamma)$. We have, [17, Prop. 2.2.1],

Proposition 1 There exists a unique connection $\widehat{\nabla}$ along $\gamma$ such that

$$
\widehat{\nabla}_{X} Y=\left(\nabla_{X} Y^{T}\right)^{T}+\left(\nabla_{X} Y^{R}\right)^{R}
$$

for any $X \in \mathfrak{X}(I)$ and $Y \in \mathfrak{X}(\gamma)$.

This connection $\widehat{\nabla}$ is called the Fermi-Walker connection of $\gamma$. It has the suggestive property that if $Y \in \mathfrak{X}(\gamma)$ satisfies $Y=Y^{R}$, then $\left(\widehat{\nabla}_{X} Y\right)_{t} \in R_{t}$ for any $t$. 2.2.2],

Denote by $\frac{\widehat{D}}{d t}$ the covariant derivative corresponding to $\widehat{\nabla}$. Then, we have $[17$, Prop.

$$
\frac{\widehat{D} Y}{d t}=\frac{D Y}{d t}+g\left(\gamma^{\prime}, Y\right) \frac{D \gamma^{\prime}}{d t}-g\left(\frac{D \gamma^{\prime}}{d t}, Y\right) \gamma^{\prime}
$$

for any $Y \in \mathfrak{X}(\gamma)$. Note that $\frac{\widehat{D}}{d t}=\frac{D}{d t}$ if and only if $\gamma$ is in free falling.

The acceleration $\frac{D \gamma^{\prime}}{d t}$ satisfies $\frac{D \gamma^{\prime}}{d t}(t) \in R_{t}$, for any $t$. Therefore, it is observed by $\gamma$ whereas the velocity $\gamma^{\prime}$ is not.

A field of observers or reference frame on the spacetime $(M, g)$ (see [14] or [17]) is a unit timelike vector field $Z \in \mathfrak{X}(M)$ pointing to the future. Note that each integral curve of $Z$ is an observer in $M$. If, in addition, $Z$ is a geodesic vector field, i.e. $\nabla_{Z} Z \equiv 0$, then every observer in $Z$ will be in free falling.

A field of observers $Z$ induces a smooth distribution on $M$, denoted by $Z^{\perp}$, which is given by the kernel of the 1 -form $Z^{b}=g(Z, \cdot)$, metrically equivalent to $Z$. When this distribution is integrable, i.e., $Z^{b} \wedge d Z^{b}=0$, then $Z$ is said to be locally synchronizable, see [14, Chap. 12] or [17, Chap. 2], and making use of the Frobenius Theorem (see [21]), we know that the spacetime $(M, g)$ is foliated by a family of spacelike hypersurfaces $\mathcal{F}=\left\{\mathcal{F}_{\lambda}\right\}$ and each leaf $\mathcal{F}_{\lambda}$ of the induced foliation $\mathcal{F}$ represents the "space" for the family of observers in $Z$. It is well-known that being locally synchronizable is equivalent to assert that each $p \in M$ has a neighborhood where $d Z^{b}=f d t$, for certain smooth functions $f>0, t$, see [17], and so, the hypersurfaces $\{t=$ constant $\}$ locally coincide with the leaves of the foliation $\mathcal{F}$. Thus, any observer may be synchronized through the "compromise time" $t$, obtained rescaling its proper time. In the more restrictive case that $Z^{b}$ is closed, i.e., $d Z^{b}=0$, it is said that the field of observer $Z$ is locally proper time synchronizable. The widely known Poincaré Lemma (see [21]) assures that any closed 1-form is locally exact, which means that it is locally the differential of a smooth function. Therefore, locally, $d Z^{b}=d t$. Hence, observers are synchronized directly by its proper time (up to a constant). 


\section{Infinitesimal relative position vector fields}

Let $(M, g)$ be a relativistic spacetime, and $Z$ a field of observers on $M$. Fix an observer $\gamma: I \longrightarrow M$ in $Z$ such that $\gamma(0)=p$, and take an orthonormal basis $\left\{e_{1}, \ldots, e_{n}\right\}$ of the Euclidean vector subspace $\left\{\gamma^{\prime}(0)\right\}^{\perp}$ of $T_{p} M$. Consider the Fermi-Walker parallel reference frame $\left\{E_{1}(t), \ldots, E_{n}(t)\right\}$ through $\gamma$, such that $E_{i}(0)=e_{i}, i=1, \ldots, n$ and define a coordinate system on a tubular neighborhood of $\gamma$,

$$
\left(t, x_{1}, \ldots, x_{n}\right) \mapsto \exp _{\gamma(t)}\left(\sum_{i=1}^{n} x_{i} E_{i}(t)\right) .
$$

This coordinate system around the observer $\gamma$ describes a new parametrization of the world lines of the observers in $Z$ nearby to $\gamma$ and, as a consequence, a new local timelike vector field $\bar{Z}$, obtained reparameterizing the local flux of $Z$, and such that $\bar{Z}_{\gamma(t)}=Z_{\gamma(t)}$. The local flux of $\bar{Z}, \Psi_{s}$, does preserve the infinitesimal restspace of the observer $\gamma$, i.e., if $v \in\left\{\gamma^{\prime}(0)\right\}^{\perp}$, then $\left.d \Psi_{t}\right|_{p}(v) \in\left\{\gamma^{\prime}(t)\right\}^{\perp}$.

Let $\left\{v_{1}, \ldots, v_{n}\right\}$ be a basis of $\left\{\gamma^{\prime}(0)\right\}^{\perp}$, therefore, we may construct the following (adapted to $\gamma$ ) coordinate system in a tubular neighborhood of $\gamma, \mathcal{V} \subseteq M$,

$$
\left(t, x_{1}, \ldots, x_{n}\right) \mapsto \exp _{\gamma(t)}\left(\sum_{i=1}^{n} x_{i} V_{i}(t)\right),
$$

where $\left\{V_{i}\right\}_{i=1}^{n}$ are the only $\bar{Z}$-Lie parallel (or $\Psi$-invariant) vector fields along $\gamma$ verifying $V_{i}(0)=v_{i}, i=1, \ldots, n$.

Now, let $\mathcal{E}$ be a sufficiently small open set around of $\mathbf{0} \in\left\{\gamma^{\prime}(0)\right\}^{\perp}$ and let $q \in \mathcal{S}_{p} \equiv$ $\exp _{p}(\mathcal{E})$ (i.e., the spacelike hypersurface $\{t=0\}$ in the adapted tubular neighborhood, which $\gamma$ perceives when its proper time is 0 ) be an event, and $\sigma$ an observer in $Z$ such that $\sigma(0)=q$. Hence, there exists a unique spacelike vector field along $\gamma, V(t)$, satisfying $\sigma(t)=\exp _{\gamma(t)}(V(t))$. This vector field will be considered the relative position vector field of the nearby observer $\sigma$ with respect to $\gamma$. Next we expose an infinitesimal formal version of this notion.

Definition 2 We define the infinitesimal relative position vector field associated to $v$ with respect to $\gamma$ as the only $\Psi$-invariant vector field along $\gamma$ with $V(0)=v$,

$$
V(t)=\left.d \Psi_{t}\right|_{\gamma(0)}(v)
$$

Equivalently, given any spacelike vector field along $\gamma, V$, we say $V$ is an infinitesimal relative position vector field for $\gamma$ if it is invariant under $\Psi$, this is, if it is Lie-parallel with respect to $\bar{Z}$, i.e, for each $t_{0}$ there exists a neighborhood of $\gamma\left(t_{0}\right)$ and a vector field in that neighborhood, $\bar{V}$, such that $L_{\bar{Z}} \bar{V}=0$ and $\bar{V}_{\mid \gamma}=V$. Notice that $V(t) \in\left(\gamma^{\prime}(t)\right)^{\perp}$ for all $t$.

Next, we proceed to characterize the infinitesimal relative position vector fields. In order to do so, we need to revisit the construction at the beginning of the section. Notice that there exists necessarily a positive smooth function on a tubular neighborhood of the observer $\gamma, h$, with $(h \circ \gamma)(t)=1, \forall t \in I$, such that

$$
\bar{Z}:=h Z \text {. }
$$


Given $v \in\left(\gamma^{\prime}(0)\right)^{\perp}$, a vector field $\bar{V}$ may be defined on a neighborhood of $\gamma(0)$ such that $\bar{V}_{\gamma(0)}=v$ and $[\bar{Z}, \bar{V}]=0$. Hence, a direct computation shows that along $\gamma$

$$
0=Z(g(\bar{V}, Z))=-\bar{V}(h)+g\left(\nabla_{Z} Z, \bar{V}\right) .
$$

And so $\nabla h_{\mid \gamma}=\nabla_{Z} Z$.

There is another natural definition to describe the relative position of nearby observers to a given one, which can be found in [17, Definition 2.3.2.].

Definition 3 Given a field of observers, $Z$, a vector field $W$ over an observer $\gamma$ is called a neighbor vector field of $\gamma$ in $Z$ if there exists a vector field $\tilde{W}$ over $\gamma$ such that $\tilde{W}^{R}=W$ and $L_{Z} \tilde{W}=0$.

It is clear that, given $v \in\left(\gamma^{\prime}(0)\right)^{\perp}$, there exists a unique neighbor vector field of $\gamma$ in $Z$, $W$, such that $W(0)=v$.

The following result shows the equivalence between the notion of infinitesimal relative position vector field and neighbor vector field.

Theorem 4 Let $Z$ be a field of observers on the spacetime $(M, g), \gamma: I \longrightarrow M$ an observer in $Z$ and $v \in\left(\gamma^{\prime}(0)\right)^{\perp}$. Consider the only neighbor vector field, $W(t)$, such that $W(0)=v$. Then, $W(t)$ is an infinitesimal relative position vector field for the observer $\gamma$.

Proof. Consider a smooth positive function on a neighborhood of the observer $\gamma, \tilde{h}$, such that $\tilde{h} \circ \gamma \equiv 1$ and $\nabla \tilde{h}_{\mid \gamma}=\nabla_{Z} Z$. Define $\tilde{Z}=\tilde{h} Z$ and consider a vector field $\tilde{V}$ such that $\tilde{V}_{\gamma(0)}=v$ and $[\tilde{Z}, \tilde{V}]=0$. Notice that $\tilde{V}_{\mid \gamma(t)} \in\left(\gamma^{\prime}(t)\right)^{\perp}$.

Let $\tilde{W}$ be a vector field defined on a neighborhood $U \subseteq M$ of $\gamma$ by $\tilde{W}=\tilde{V}+f Z$, with $f \in C^{\infty}(U)$.

$$
[Z, \tilde{W}]=[Z, \tilde{V}]+Z(f) Z
$$

Since,

$$
0=[\tilde{Z}, \tilde{V}]=\tilde{h}[Z, \tilde{V}]-\tilde{V}(\tilde{h}) Z,
$$

we get

$$
[Z, \tilde{W}]=\tilde{V}(\ln (\tilde{h})) Z+Z(f) Z .
$$

We only need to chose $f$ such that $Z(f)=-\tilde{V}(\ln (\tilde{h}))$, and the uniqueness of $W$ assures that $\tilde{V}_{\mid \gamma}=W$. In particular, when $\tilde{h}=h$, we deduce that $W$ is an infinitesimal relative position vector field for the observer $\gamma$.

Notice that the proof above gives us an alternative definition of relative position vector fields.

Corollary 5 Let $Z$ be a field of observers on the spacetime $(M, g), \gamma: I \longrightarrow M$ an observer in $Z$ and $\tilde{h}$ a smooth positive function defined on a tubular neighborhood of $\gamma$. Then, the flux of $\tilde{h} Z$ defines an infinitesimal relative position vector field with respect to $\gamma$ if and only if the function $\tilde{h}$ satisfies,

(a) $(\tilde{h} \circ \gamma)(t)=1, \quad \forall t \in I$.

(b) $\left.\nabla \tilde{h}\right|_{\gamma(t)}=\left.\nabla_{\gamma^{\prime}(t)} Z\right|_{\gamma(t)}, \quad \forall t \in I$. 
Taking into account the previous theorem, the Fermi-Walker derivative $\frac{\widehat{D} V}{d t}$ of an infinitesimal relative position vector field $V \in \mathfrak{X}(\gamma)$ represents the velocity of nearby neighboring observers in $Z$ with respect to the observer $\gamma$, i.e., the velocity measured by the observer $\gamma$.

The Fermi-Walker derivative of an infinitesimal relative position vector field admits another geometrical interesting expression, as it follows from the next result. This proposition can be found in [17, Proposition 2.3.4.] in terms of neighbor vector fields with a longer proof.

Proposition 6 Given a field of observers $Z$ in the relativistic spacetime $(M, g)$, and $\gamma$ an observer in $Z$, for any infinitesimal relative position vector field $V \in \mathfrak{X}(\gamma)$, the following identity holds

$$
\frac{\widehat{D} V}{d t}=-A_{Z}^{\prime}(V):=\nabla_{V} Z
$$

Proof. By using that $\bar{Z}=h Z, h \circ \gamma \equiv 1, \frac{\widehat{D} V}{d t} \perp Z$ and $L_{\bar{Z}} V=0$, we get

$$
g\left(\frac{\widehat{D} V}{d t}, U\right)=g\left(\nabla_{Z} V, U\right)=g\left(\nabla_{\bar{Z}} \bar{V}, U\right)=g\left(\nabla_{\bar{V}} \bar{Z}, U\right)=g\left(\nabla_{V} Z, U\right),
$$

for any $U \in \mathfrak{X}(\gamma)$ orthogonal to $Z_{\gamma(t)}$.

Next, we analyze the geometry of the linear operator $A_{Z}^{\prime}: \Gamma\left(Z^{\perp}\right) \subset \Gamma(T M) \longrightarrow \Gamma\left(Z^{\perp}\right)$, defined in the previous proposition. This linear operator may be decomposed in its symmetric $\widehat{S}$ and skew-symmetric $\widehat{\omega}$ parts,

$$
-A_{Z}^{\prime}=\widehat{S}+\widehat{\omega}
$$

where $\widehat{S}$ is self-adjoint for $g$, and $\widehat{\omega}$ skew-adjoint. Denote by $S$ and $\omega$ the corresponding fields of 2-covariant associated tensors,

$$
\begin{gathered}
S(V, W)=g(\widehat{S}(V), W)=\frac{1}{2}\left(g\left(\nabla_{V} Z, W\right)+g\left(\nabla_{W} Z, V\right)\right), \\
\omega(V, W)=g(\widehat{\omega}(V), W)=\frac{1}{2} \operatorname{Curl}(Z)(V, W),
\end{gathered}
$$

where $V, W$ are spacelike vector fields in $Z^{\perp}$, and $\omega$ is the vorticity or Coriolis tensor field. The name "vorticity" means that, if the observers in $Z$ represent the word lines of the particles of a fluid and $\gamma$ is the trajectory of one of them, $\omega\left(\gamma^{\prime}\right)$ gives certain measure about how $\gamma$ see the others turn around. Notice that $\omega=\frac{1}{2} \operatorname{Curl}(Z)$ can also be expressed as $\omega=d Z^{b}$, being $Z^{b}=g(Z, \cdot)$ the 1-form metrically equivalent to $Z$.

On the other hand, the symmetric operator $\widehat{S}$ can be decomposed as

$$
\widehat{S}=\frac{\operatorname{div}(Z)}{n} I+\Theta
$$

where $I$ denotes the identity endomorphism of $\Gamma\left(Z^{\perp}\right), \Theta$ is the traceless part of $\widehat{S}$ (the shear tensor), and $\theta(Z)=\operatorname{div}(Z)$. The term $\frac{\operatorname{div}(Z)}{n} I$ represents the expansion or contraction, i.e., fixed an observer in $Z$, it measures how nearby neighboring observers go away on average, while $\Theta$ measures the deviations of this average. 


\section{Irrotational and spatially conformal Killing fields of observers}

We will begin this section recalling some basic definitions.

A field of observers $Z$ is said to be irrotational or vorticity-free if $\widehat{\omega}=0$, i.e.,

$$
g\left(\nabla_{V} Z, W\right)=g\left(\nabla_{W} Z, V\right), \quad \forall V, W \in \Gamma\left(Z^{\perp}\right) .
$$

It seems natural to think that an observer $\gamma$ in $\mathrm{Z}$ will affirm that his neighboring observers do not rotate if the Fermi-Walker derivative of each infinitesimal relative position vector field on $\gamma$ is proportional to the infinitesimal relative position vector field itself. As it is immediate to show, this condition assures the irrotational character of $Z$. Nevertheless, if a field of observers $Z$ is irrotational, it is not possible in general to assure the condition of collinearity on the Fermi-Walker derivative. Indeed,

Example 7 Condition (6) can not be derived from the irrotational character of $Z$. For instance, let us consider the Lorentz-Minkowski spacetime $\mathbb{L}^{3}$ with its standard coordinates $(u, x, y)$. Take the field of observers $Z=\sqrt{1+u^{2}} \partial_{u}+u \partial_{x}$. Any vector field orthogonal to $Z$ takes the form

$$
V=\frac{u V_{1}}{\sqrt{1+u^{2}}} \partial_{u}+V_{1} \partial_{x}+V_{2} \partial_{y}
$$

for arbitrary smooth functions $V_{1}, V_{2}$. We can compute

$$
\left\langle\nabla_{V} Z, W\right\rangle=\frac{u}{\left(1+u^{2}\right)^{3 / 2}} V_{1} W_{1},
$$

for any $V, W$ orthogonal to $Z$. From which we get that $Z$ is irrotational.

Now, we fix $p \in \mathbb{L}^{3}$ and we denote by $E_{1}$ and $E_{2}$ the infinitesimal relative position vector fields along the integral curve of $Z$ through $p$ such that $E_{1}(p)=\frac{u}{\sqrt{1+u^{2}}} \partial_{u}(p)+\partial_{x}(p)$ and $E_{2}(p)=\partial_{y}(p)$. Then,

$$
\frac{\widehat{D} E_{1}}{d u}(p)=\frac{u}{\left(1+u^{2}\right)^{3 / 2}} E_{1}(p), \quad \text { while } \quad \frac{\widehat{D} E_{2}}{d u}(p)=0 .
$$

Definition 8 A field of observers $Z$ is spatially conformal Killing or shear-free if there exists a function $\lambda \in C^{\infty}(M)$, such that

$$
g\left(\nabla_{V} Z, W\right)+g\left(\nabla_{W} Z, V\right)=2 \lambda g(V, W), \quad \forall V, W \in \Gamma\left(Z^{\perp}\right) .
$$

Therefore $\widehat{S}=\lambda I$, with $\lambda=\frac{1}{n} \operatorname{div}(Z)$, i.e., the shear tensor of $Z$ is identically zero. When the function $\lambda$ is identically zero, the field $Z$ is called spatially Killing or spatially rigid.

In the case that $Z$ satisfies the condition $L_{Z} g=2 \lambda g$, or equivalently

$$
g\left(\nabla_{X} Z, Y\right)+g\left(\nabla_{Y} Z, X\right)=2 \lambda g(X, Y), \quad \forall X, Y \in \Gamma(T M),
$$

the field of observers $Z$ is called conformal Killing.

Now, taking into account Equation (2), we conclude that, 
Proposition 9 Let $Z$ be a field of observers in a spacetime $(M, g)$. Then, $Z$ is irrotational and spatially conformal Killing if and only if

$$
\frac{\widehat{D} V}{d t}=\frac{\operatorname{div}(Z)}{n} V
$$

for any infinitesimal relative position vector field $V \in \mathfrak{X}(\gamma)$, and any observer $\gamma$ in $Z$. Analogously, the field of observers $Z$ will be spatially rigid and irrotational if and only if

$$
\frac{\widehat{D} V}{d t}=0
$$

for any infinitesimal relative position vector field $V \in \mathfrak{X}(\gamma)$, being $\gamma$ an arbitrary observer in $Z$.

The importance of conformally stationary spacetimes has been widely described in Section 1. Observe that if $(M, g)$ is a conformally stationary spacetime whose timelike conformal Killing vector field $X$ is irrotational, then it is not difficult to see that the field of observers $\frac{X}{\|X\|}$ determined by $X$ is irrotational and spatially conformal Killing.

The following question arises naturally: if $Z$ is an irrotational and spatially conformal Killing vector field of observers in a spacetime $(M, g)$, under which conditions on the geometry and topology of $M$ and on the field of observers $Z$, does a function $\varphi$ exist such that the vector field $\varphi Z$ is a conformal Killing vector field?

We are interested in the case in which the function $\varphi$ has no zeros, and consequently the spacetime will be conformally stationary.

Suppose that $Z$ satisfies condition (6). It is clear that $\varphi Z$ is irrotational and spatially conformal Killing for any $\varphi>0$, with conformal factor $\varphi \frac{\operatorname{div}(Z)}{n}$. Let $\gamma$ be an observer in $Z$, and $v \in\left(\gamma^{\prime}(t)\right)^{\perp}$. We have

$$
\begin{gathered}
g\left(\nabla_{v}(\varphi Z), \gamma^{\prime}(t)\right)+g\left(\nabla_{\gamma^{\prime}(t)}(\varphi Z), v\right)=-v(\varphi)+\varphi g\left(\nabla_{\gamma^{\prime}(t)} Z, v\right), \\
g\left(\nabla_{\gamma^{\prime}(t)}(\varphi Z), \gamma^{\prime}(t)\right)=-\gamma^{\prime}(t)(\varphi) .
\end{gathered}
$$

From (5), we deduce that $\varphi$ must verify

$$
v(\log (\varphi))=g\left(\nabla_{\gamma^{\prime}(t)} Z, v\right), \quad \text { and } \quad Z(\log (\varphi))=\frac{\operatorname{div}(Z)}{n},
$$

for any $v \in\left(Z_{\gamma(t)}\right)^{\perp}$. Both conditions may be summarized as

$$
\nabla \log (\varphi)=\nabla_{Z} Z-\frac{\operatorname{div}(Z)}{n} Z .
$$

Hence, from a direct application of the Poincaré Lemma (see [21]), we get the following result.

Theorem 10 Let $Z$ be a field of observers in a spacetime $\left(M^{n+1}, g\right)$ such that condition (6) holds for any observer $\gamma$ in $Z$ and for every infinitesimal relative position vector field $V \in \mathfrak{X}(\gamma)$. 
(a) If the following condition holds

$$
\operatorname{Curl}\left(\nabla_{Z} Z-\frac{1}{n} \operatorname{div}(Z) Z\right)=0,
$$

then for each point $p \in M$ there exists a neighborhood $U_{p}$ and a positive function $\varphi \in$ $C^{\infty}\left(U_{p}\right)$ such that $\varphi Z$ is conformal Killing.

(b) If in addition $M$ is contractible to a point, then the function $\varphi$ is globally defined.

(c) If there exists a positive function $\varphi \in C^{\infty}(M)$ such that $\varphi Z$ is conformal Killing, then (12) is satisfied.

In addition, note that if (12) holds, from (10) we have

$$
\varphi(\gamma(t))=\varphi(\gamma(0)) \exp \left(\frac{1}{n} \int_{\gamma} \operatorname{div}(Z)\right)
$$

for each observer $\gamma$ in $Z$. Thus, if we know the function $\varphi$ on a hypersurface orthogonal to $Z$ passing through a point $p$, we also know the function $\varphi$ on a neighborhood of $p$.

Finally, we assume that $\frac{\widehat{D} V}{d t}=0$ for any observer $\gamma$ in $Z$ and for any infinitesimal relative position vector field $V \in \mathfrak{X}(\gamma)$. We know that $Z$ is irrotational and spatially rigid, but, what must $Z$ satisfy so that $\varphi Z$ is Killing for some positive function $\varphi$ ? The answer is contained in the following corollary of Th.10.

Theorem 11 Let $Z$ be a field of observers in a spacetime $(M, g)$ such that $\frac{\widehat{D} V}{d t}=0$ for any observer $\gamma$ in $Z$ and for every infinitesimal relative position vector field $V \in \mathfrak{X}(\gamma)$.

(a) If the following condition holds

$$
\operatorname{Curl}\left(\nabla_{Z} Z\right)=0
$$

then for each point $p \in M$ there exists a neighborhood $U_{p}$ and a positive function $\varphi \in$ $C^{\infty}\left(U_{p}\right)$ such that $\varphi Z$ is Killing.

(b) If in addition $M$ is contractible to a point, then the function $\varphi$ is globally defined.

(c) If there exists a positive function $\varphi \in C^{\infty}(M)$ such that $\varphi Z$ is Killing, then (13) is satisfied.

Remark 12 Notice that the vector field $\nabla_{Z} Z$ represents the proper acceleration of each observer in $Z$. In fact, condition (13) assures that this vector field must be locally a gradient vector field. Obviously, when the manifold $M$ is simply-connected, $\nabla_{Z} Z$ is globally a gradient vector field.

Remark 13 Given an observer $\gamma$ in $Z$ and an infinitesimal relative position vector field $V \in \mathfrak{X}(\gamma)$, a straightforward computation shows that condition (13) is satisfied if and only if $\nabla_{Z} Z$ is irrotational and $g\left(\nabla_{Z} Z, V\right)$ is constant along the world line of $\gamma$ for all relative position vector field $V$. 


\section{Characterizations of GRW spacetimes}

There is a particularly interesting class of spacetimes admitting an irrotational and spatially conformal Killing field of observers, the Generalized Robertson-Walker (GRW) spacetimes. A GRW spacetime with base $\left(I,-d t^{2}\right)$, fiber $\left(F, g_{F}\right)$ and warping function $f$ is the product manifold $M=I \times F$ endowed with the Lorentzian metric

$$
g^{f}=-d t^{2}+f^{2}(t) g_{F},
$$

see [1]. GRW spacetimes are particular cases of warped products, which have been widely used in general relativity (see Introduction). For any GRW spacetime, the vector field $\partial_{t}$ is a field of observers which is geodesic (the observers in $Z$ are in free falling), irrotational, spatially conformal Killing and divergence non-spacelike depending, see [18]. Indeed, given a simply connected relativistic spacetime $(M, g)$ with a complete vector field of observers $Z$ satisfying the previously cited properties, then $(M, g)$ is (globally) a GRW spacetime with $\partial_{t}=Z$, [18, Theorem 2.1]. If the global hypotheses (simply connection of $M$ and completeness) are removed, then $(M, g)$ is locally a GRW spacetime. From this results together with Proposition 9 we get the following theorem.

Theorem 14 Let $(M, g)$ be a simply connected relativistic spacetime with a complete vector field of observers $Z$ and suppose that the observer in $Z$ are in free falling, the gradient of $\operatorname{div}(Z)$ is pointwise parallel to $Z$ and the Fermi-Walker derivative of any infinitesimal relative position vector field along any of its integral curves is proportional to the infinitesimal relative position vector field itself. Then $(M, g)$ is (globally) a GRW spacetime with $\partial_{t}=Z$. If $M$ is not asked to be simply connected and $Z$ is not needed to be complete, then $(M, g)$ is locally a GRW spacetime.

This last result constitutes a new characterization of GRW spacetimes through the behavior of the infinitesimal relative position vector fields associated to certain class of observers in them. Others recent characterizations of this relevant family of spacetimes can be found in [4], [10], and [12] and references therein.

\section{References}

[1] L.J. Alías, A. Romero, and M. Sánchez. Uniqueness of complete spacelike hypersurfaces of constant mean curvature in generalized Robertson-Walker spacetimes. Gen. Relativity Gravitation, 27(1):71-84, 1995.

[2] J.K. Beem, P.E. Ehrlich, and K.L. Easley. Global Lorentzian Geometry, volume 202 of Pure and Applied Mathematics. Marcel Dekker, New York, 1996.

[3] M. Caballero, A. Romero, and R.M. Rubio. Constant mean curvature spacelike hypersurfaces in lorentzian manifolds with a timelike gradient conformal vector field. Classical and Quantum Gravity, 28:145009, 2011.

[4] B-Y. Chen. A simple characterization of generalized Robertson-Walker spacetimes. Gen. Rel. Grav., 46(12):1833, 2014. 
[5] A.A. Coley and B.O.J. Tupper. Zero-curvature Friedmann-Robertson-Walker models as exact viscous magnetohydrodynamic cosmologies. The Astrophysical Journal, 271:1-8, 071983.

[6] V. Daftardar and N. Dadhich. Gradient conformal killing vectors and exact solutions. General Relativity and Gravitation, 26(9):859-868, Sep 1994.

[7] K.L. Duggal and R. Sharma. Connection and Curvature Symmetries, pages 134-155. Springer US, Boston, MA, 1999.

[8] D. Eardley, J. Isenberg, J. Marsden, and V. Moncrief. Homothetic and conformal symmetries of solutions to Einstein's equations. Communications in Mathematical Physics, 106(1):137-158, Mar 1986.

[9] J.J. Ferrando, J.A. Morales, and M. Portilla. Inhomogeneous space-times admitting isotropic radiation: Vorticity-free case. Phys. Rev. D, 46:578-584, Jul 1992.

[10] M. Gutiérrez and B. Olea. Global decomposition of a Lorentzian manifold as a GRW spacetime. Differential Geometry and its Applications, 27:146-156, 022009.

[11] S. W. Hawking. The existence of cosmic time functions. Proceedings of the Royal Society of London. Series A, Mathematical and Physical Sciences, 308(1494):433-435, 1969.

[12] C. A. Mantica and L. G. Molinari. Generalized Robertson-Walker space times, a survey. International Journal of Geometric Methods in Modern Physics, 14:1730001, 032017.

[13] L. G. Molinari and C. A. Mantica. A simple property of the weyl tensor for a shear, vorticity and acceleration-free velocity field. General Relativity and Gravitation, 50(7):81, Jun 2018.

[14] B. O'Neill. Semi-Riemannian Geometry With Applications to Relativity, 103, Volume 103 (Pure and Applied Mathematics). Academic Press, 1983.

[15] M. Rainer and H-J. Schmidt. Inhomogeneous cosmological models with homogeneous inner hypersurface geometry. General Relativity and Gravitation, 27(12):1265-1293, Dec 1995.

[16] M. J. Rebouças, J. E. F. Skea, and R. K. Tavakol. Cosmological models expressible as gradient vector fields. Journal of Mathematical Physics, 37(2):858-873, 1996.

[17] R. K. Sachs and H. Wu. General Relativity for Mathematicians. Grad. Texts Math. 48. Springer-Verlag, 1977.

[18] M. Sánchez. On the geometry of generalized robertson-walker spacetimes: Geodesics. General Relativity and Gravitation, 30(6):915-932, Jun 1998.

[19] Hans Stephani, Dietrich Kramer, Malcolm MacCallum, Cornelius Hoenselaers, and Eduard Herlt. Exact Solutions of Einstein's Field Equations. Cambridge Monographs on Mathematical Physics. Cambridge University Press, 2 edition, 2003.

[20] R. Treciokas and G. F. R. Ellis. Isotropic solutions of the Einstein-Boltzmann equations. Comm. Math. Phys., 23(1):1-22, 1971. 
[21] R.W. Warner. Foundations of Differentiable Manifolds and Lie Groups. Springer-Verlag, 1983.

[22] E. Zafiris. Irreducible Decomposition of Einstein's Equations in Spacetimes with Symmetries. Annals of Physics, 263:155-178, March 1998. 